\title{
Liraglutide for non-alcoholic fatty liver disease in children and adolescents: has a new era arrived?
}

\author{
Dimitrios loannis Patoulias \\ First Department of Internal Medicine, General Hospital “Hippokration”, Thessaloniki, Greece
}

Gastroenterology Rev 2019; 14 (3): 221-222

DOI: https://doi.org/10.5114/pg.2019.88172

Address for correspondence: Dimitrios loannis Patoulias MD, First Department of Internal Medicine, General Hospital "Hippokration", Thessaloniki, Greece, phone: +30 6946900777, e-mail: dipatoulias@gmail.com

Liraglutide, an injectable glucagon-like peptide-1 receptor agonist (GLP-1RA), has been approved for use in adult patients with diabetes since 2010. There is an ongoing discussion regarding the potential role of liraglutide in the treatment of non-alcoholic fatty liver disease (NAFLD) in the paediatric population [1, 2]. To date, no solid evidence regarding any therapy of this disease exists, and thus no medication is currently recommended [3].

In the hallmark Liraglutide Efficacy and Action in NASH (LEAN) study, Armstrong et al. demonstrated that a significantly higher proportion of enrolled patients with non-alcoholic steatohepatitis (NASH) receiving liraglutide $1.8 \mathrm{mg}$ daily, compared to those receiving placebo (relative risk $(R R)=4.5,95 \% \mathrm{Cl}: 1.1-18.9)$, achieved the primary efficacy outcome, which was defined as the resolution of steatohepatitis (disappearance of hepatocyte ballooning) without worsening of fibrosis [4]. Concerning secondary efficacy outcomes, it was also shown that liraglutide induced a significant weight loss compared to placebo, equal to $4.39 \mathrm{~kg}$ [4]. Induction of delay in gastric emptying and increased satiation, as measured by maximum tolerated gastric volume, mediate the weight loss seen in patients treated with liraglutide [5]. A decrease in fasting hunger and energy intake and an increase in energy expenditure also characterise the liraglutide-induced weight loss [6].

The question that arises is whether liraglutide is a safe treatment option for paediatric patients. In a randomised, double-blind, placebo-controlled trial enrolling obese adolescents aged 12-17 years, Danne et al. demonstrated that there were no severe or serious treatment adverse events with liraglutide and no cases of treatment discontinuation, while liraglutide was effective, inducing an average weight loss equal to $2.55 \mathrm{~kg}$ [7]. However, there was a greater incidence of minor, symptomatic hypoglycaemic episodes and transient gastrointestinal adverse events among adolescents who were administered liraglutide. The pharmacokinetic profile was similar to that observed in adult patients [7]. Similar results were retrieved from another, recently published, randomised, double-blind, placebo-controlled trial enrolling obese children aged 7-11 years [8]. Mastrandrea et al. showed that liraglutide did not induce severe or serious adverse events, while it was more frequently associated with mild gastrointestinal adverse events. A few asymptomatic hypoglycaemic episodes were also reported with liraglutide, which did not require any additional treatment. A significant reduction in body mass index (BMI) z score and a non-significant reduction in body weight were observed among liraglutide-treated children. Finally, the pharmacokinetic profile of this GLP-1RA was consistent with dose proportionality [8], confirming previously published results [9].

Besides body weight loss, it seems that liraglutide can directly affect pathophysiologic mechanisms of insulin resistance (IR). In their observational study, Zhou et al. demonstrated that combined intervention with liraglutide and lifestyle modification in children with pre-diabetes compared to lifestyle modification alone resulted in a significant decrease in the homeostasis model for assessment of insulin resistance (HOMA-IR) within three months, besides significant improvement in BMI and lipid profile [10]. A significant amount of interest also lies on the results of the Ellipse trial (NCT01541215), a randomised, double-blind, placebo-controlled trial, assessing the efficacy and safety of liraglutide in combination with metformin compared to metformin alone, in children and adolescents with type 2 diabetes, which has not been published yet [11]. The effect of liraglutide on significant cardio-metabolic risk factors, directly associated with NAFLD, is due to be documented. Based on the fact that lipotoxicity and IR play a crucial role in the development of NAFLD [1], it is 
therefore deduced that they should constitute primary treatment targets.

Overall, despite the fact that there are a limited number of studies with small sample sizes assessing the efficacy and safety of liraglutide in paediatric patients, and the fact that none of the aforementioned studies evaluated measures of NAFLD and its activity (if present), in my opinion, liraglutide might evolve as a promising treatment option in paediatric NAFLD and its related co-morbidities in the coming years. Larger, prospective, randomised controlled trials are required in order to elucidate this hypothesis.

\section{Conflict of interest}

The authors declare no conflict of interest.

\section{References}

1. Flisiak-Jackiewicz M, Lebensztejn D. Update on pathogenesis, diagnostics and therapy of nonalcoholic fatty liver disease in children. Clin Exp Hepatol 2019; 5: 11-21.

2. Draijer L, Benninga M, Koot B. Pediatric NAFLD: an overview and recent developments in diagnostics and treatment. Expert Rev Gastroenterol Hepatol 2019; 13: 447-61.

3. Shapiro WL, Yu EL, Arin JC, et al. Clinical practice approach to nonalcoholic fatty liver disease by pediatric gastroenterologists in the United States. J Pediatr Gastroenterol Nutr 2019; 68: 182-9.

4. Armstrong MJ, Gaunt P, Aithal GP, et al. Liraglutide safety and efficacy in patients with non-alcoholic steatohepatitis (LEAN): a multicentre, double-blind, randomised, placebo-controlled phase 2 study. Lancet 2016; 387: 679-90.

5. Halawi H, Khemani D, Eckert D, et al. Effects of liraglutide on weight, satiation, and gastric functions in obesity: a randomised, placebo-controlled pilot trial. Lancet Gastroenterol Hepatol 2017; 2: 890-9.

6. Horowitz M, Flint A, Jones KL, et al. Effect of the once-daily human GLP-1 analogue liraglutide on appetite, energy intake, energy expenditure and gastric emptying in type 2 diabetes. Diabetes Res Clin Pract 2012; 97: 258-66.

7. Danne T, Biester T, Kapitzke K, et al. Liraglutide in an adolescent population with obesity: a randomized, double-blind, placebo-controlled 5-week trial to assess safety, tolerability, and pharmacokinetics of liraglutide in adolescents aged 12-17 years. J Pediatr 2017; 181: 146-53.e3.

8. Mastrandrea LD, Witten L, Carlsson Petri KC, et al. Liraglutide effects in a paediatric (7-11 y) population with obesity: a randomized, double-blind, placebo-controlled, short-term trial to assess safety, tolerability, pharmacokinetics, and pharmacodynamics. Pediatr Obes 2019; 14: e12495.

9. Petri KC, Jacobsen LV, Klein DJ. Comparable liraglutide pharmacokinetics in pediatric and adult populations with type 2 diabetes: a population pharmacokinetic analysis. Clin Pharmacokinet 2015; 54: 663-70.

10. Zhou QX, Wang ZY, Zhao HF, Wang S. The effects of GLP-1 analogues on pre-diabetes of the children. Exp Ther Med 2017; 13: $1426-30$
11. Accessed online at: https://clinicaltrials.gov/ct2/show/ NCT01541215.

Received: 24.03.2019

Accepted: 26.03.2019 